\title{
Association of plasma and CSF cytochrome P450, soluble epoxide hydrolase, and ethanolamide metabolism with Alzheimer's disease
}

Kamil Borkowski ${ }^{1 *}$ (D, Theresa L. Pedersen², Nicholas T. Seyfried ${ }^{3}$, James J. Lah ${ }^{4}$, Allan I. Levey ${ }^{4}$, Chadwick M. Hales ${ }^{4}$, Eric B. Dammer ${ }^{3}$, Colette Blach ${ }^{5}$, Gregory Louie ${ }^{6}$, Rima Kaddurah-Daouk, John W. Newman ${ }^{1,8,9}$ and Alzheimer's

Disease Metabolomics Consortium

\begin{abstract}
Background: Alzheimer's disease, cardiovascular disease, and other cardiometabolic disorders may share inflammatory origins. Lipid mediators, including oxylipins, endocannabinoids, bile acids, and steroids, regulate inflammation, energy metabolism, and cell proliferation with well-established involvement in cardiometabolic diseases. However, their role in Alzheimer's disease is poorly understood. Here, we describe the analysis of plasma and cerebrospinal fluid lipid mediators in a case-control comparison of 150 individuals with Alzheimer's disease and 135 healthy controls, to investigate this knowledge gap.

Methods: Lipid mediators were measured using targeted quantitative mass spectrometry. Data were analyzed using the analysis of covariates, adjusting for sex, age, and ethnicity. Partial least square discriminant analysis identified plasma and cerebrospinal fluid lipid mediator discriminates of Alzheimer's disease. Alzheimer's disease predictive models were constructed using machine learning combined with stepwise logistic regression.

Results: In both plasma and cerebrospinal fluid, individuals with Alzheimer's disease had elevated cytochrome P450/soluble epoxide hydrolase pathway components and decreased fatty acid ethanolamides compared to healthy controls. Circulating metabolites of soluble epoxide hydrolase and ethanolamides provide Alzheimer's disease predictors with areas under receiver operator characteristic curves ranging from 0.82 to 0.92 for cerebrospinal fluid and plasma metabolites, respectively.
\end{abstract}

Conclusions: Previous studies report Alzheimer's disease-associated soluble epoxide hydrolase upregulation in the brain and that endocannabinoid metabolism provides an adaptive response to neuroinflammation. This study supports the involvement of P450-dependent and endocannabinoid metabolism in Alzheimer's disease. The results further suggest that combined pharmacological intervention targeting both metabolic pathways may have therapeutic benefits for Alzheimer's disease.

Keywords: Alzheimer's disease, Lipid mediators, Oxylipins, Endocannabinoids, Cognition

\footnotetext{
* Correspondence: kborkowski@ucdavis.edu

'West Coast Metabolomics Center, Genome Center, University of California Davis, Davis, CA 95616, USA

Full list of author information is available at the end of the article
}

(c) The Author(s). 2021 Open Access This article is licensed under a Creative Commons Attribution 4.0 International License, which permits use, sharing, adaptation, distribution and reproduction in any medium or format, as long as you give appropriate credit to the original author(s) and the source, provide a link to the Creative Commons licence, and indicate if changes were made. The images or other third party material in this article are included in the article's Creative Commons licence, unless indicated otherwise in a credit line to the material. If material is not included in the article's Creative Commons licence and your intended use is not permitted by statutory regulation or exceeds the permitted use, you will need to obtain permission directly from the copyright holder. To view a copy of this licence, visit http://creativecommons.org/licenses/by/4.0/ The Creative Commons Public Domain Dedication waiver (http://creativecommons.org/publicdomain/zero/1.0/) applies to the data made available in this article, unless otherwise stated in a credit line to the data. 


\section{Introduction}

Risk factors for Alzheimer's disease (AD) include cardiometabolic disorders, low-grade systemic inflammation, and altered lipid and energy metabolism [1-3]. Lipid mediators are lipid-derived signaling molecules that regulate both acute and low-grade systemic inflammation and energy metabolism along with other processes [4]. Circulating lipid mediators have been implicated in the pathogenesis of cardiometabolic diseases [5-7]; however, their involvement in $\mathrm{AD}$ is still not well described.

Four important families of such lipid mediators readily detected in the circulation are the oxygenated polyunsaturated fatty acids (i.e., oxylipins), the endogenous cannabinoid receptor activators and their structural equivalents (i.e., endocannabinoids, including monoacylglycerols and ethanolamides), bile acids, and steroids. Oxylipins are mainly oxygenated products of polyunsaturated fatty acids (PUFAs) generated via 4 main pathways: cyclooxygenases (COX), generating mainly prostaglandins; lipoxygenases (LOX), generating mainly hydroxy-fatty acids, including pro-inflammatory leukotrienes and pro-resolving lipoxins, resolvins, and maresins [8]; cytochrome P450 (CYP) and soluble epoxide hydrolase $(\mathrm{sEH})$, generating epoxy-fatty acids and dihydroxy fatty acids, respectively [4]; and reactive oxygenmediated formation of prostanoids and hydroxy-fatty acids [9]. Products from these pathways exhibit both pro- and anti-inflammatory actions [4]. Endocannabinoids are mainly fatty acids esters and amides, which are ligands for the cannabinoid receptors $\mathrm{CB} 1$ and $\mathrm{CB} 2$, the transient potential vanilloid receptor TRPV1, and Gprotein-coupled receptor GPR55 [10], all highly expressed in the central nervous system [11]. Endocannabinoids regulate energy metabolism [10] and are generally considered anti-inflammatory [12]. Bile acids (BAs) are generated by the liver as primary bile acids and secreted into the gut to aid in lipid digestion, and they are further metabolized by the gut microbiome to form secondary bile acids [13]. After reabsorption from the gut into the blood stream, BAs regulate energy metabolism with different potency between primary and secondary species [14].

Changes in circulating lipid mediators in relation to AD were previously reported. Particularly, several oxylipins of the acute inflammation pathway were reportedly elevated in $\mathrm{AD}[15,16]$ and pro-resolving (quenching activated inflammatory signal) lipid mediators have been suggested as potential treatment for AD [17]. Specific changes in bile acid metabolism, including a decrease in primary and an increase in secondary metabolites, were also observed in AD subjects [18], and differences in bile acid clearance for cholesterol pathway were reported in AD [19]. Notably, bile acids and some steroids manifest neuroprotective functions through activation of steroid receptors [20]. However, a comprehensive analysis of the AD-related changes in circulating lipid mediators is lacking and reports of lipid mediators in cerebrospinal fluid (CSF) are minimal. In the current work, we utilize plasma and CSF samples of AD patients and cognitively normal controls from the Emory Goizueta Alzheimer's Disease Research Center (ADRC). Using a case-control approach, we provide a comprehensive analysis of $\mathrm{AD}$ associated changes in both circulating and CSF lipid mediators, including major classes of oxylipins, endocannabinoids, bile acids, and some steroids, covering multiple aspects of inflammatory cascades and regulators of energy metabolism. Moreover, to minimalize the bias caused by postprandial fluctuation in plasma lipids, the fasting state of opportunistically collected samples was estimated using a novel predictive model that uses levels of circulating lipid mediators [21].

\section{Materials and methods Subjects}

All participants from whom plasma and CSF samples were collected provided informed consent under protocols approved by the Institutional Review Board at Emory University. Cohorts included the Emory Healthy Brain Study (IRB00080300), Cognitive Neurology Research (IRB00078273), and Memory @ Emory (IRB00079069). All protocols were reviewed and approved by the Emory University Institutional Review Board. All patients received standardized cognitive assessments (including Montreal Cognitive Assessment (MoCA)) in the Emory Cognitive Neurology clinic, the ADRC, and affiliated Emory Healthy Brain Study (EHBS) [22]. All diagnostic data were supplied by the ADRC and the Emory Cognitive Neurology Program. CSF was collected by lumbar puncture and banked according to 2014 ADC/NIA best practice guidelines. All CSF samples collected from research participants in the ADRC, Emory Healthy Brain Study, and Cognitive Neurology clinic were assayed using the INNO-BIA AlzBio3 Luminex assay at AKESOgen (Peachtree Corners, GA). AD cases and healthy individuals were defined using established biomarker cutoff criteria for $\mathrm{AD}$ for each assay platform [23, 24]. In total, plasma samples were available for $148 \mathrm{AD}$ patients and 133 healthy controls and CSF samples were available for $150 \mathrm{AD}$ patients and 139 healthy controls. Plasma and CSF sample collection overlap (both plasma and CSF collected at the same day) was 145 for the AD group and 133 for the control group. Cohort summary statistics for gender, age, MoCA, AB42, tTau, pTau, ApoE genotype, and ethnicity are provided in Table S1. 


\section{Quantification of lipid mediators}

Plasma concentrations of non-esterified PUFA, oxylipins, endocannabinoids, a group of non-steroidal antiinflammatory drugs (NSAIDs) including ibuprofen, naproxen, acetaminophen, a suite of conjugated and unconjugated bile acids, and a series of glucocorticoids, progestins, and testosterone were quantified in $50 \mu \mathrm{L}$ of plasma by liquid chromatography tandem mass spectrometry (LC-MS/MS) after protein precipitation in the presence of deuterated metabolite analogs (i.e., analytical surrogates) [25]. CSF analyses were performed with 100$\mu \mathrm{L}$ samples prepared as previously reported for the analyses of sweat [26] and analyzed as reported for plasma. All samples were processed with rigorous quality control measures including case/control randomization, and the analysis of batch blanks, pooled matrix replicates, and NIST Standard Reference Material 1950 - Metabolites in Human Plasma (Sigma-Aldrich, St Louis, MO). Extraction batches were re-randomized for acquisition, with method blanks and reference materials and calibration solutions scattered regularly throughout the set. Instrument limits of detection (LODs) and limits of quantification (LOQs) were estimated according to the Environmental Protection Agency method (40 CFR, Appendix B to Part 136 revision 1.11, U.S. and EPA 821-R16-006 Revision 2). These values were then transformed into sample nanomolar concentrations by multiplying the calculated concentration by the final sample volume and dividing by the volume of sample extracted. A complete analyte list with plasma LODs and LOQs has been reported [25]. The majority of analytes were quantified against analytical standards with the exception of eicosapentaenoyl ethanolamide (EPEA), palmitoleoyl ethanolamide (POEA), and the measured PUFAs [i.e., linoleic acid (LA), alpha-linolenic acid (aLA), arachidonic acid (AA), eicosapentaenoic acid (EPA), docosahexaenoic acid (DHA)]. For these compounds, area counts were recorded and adjusted for deuteratedsurrogate responses, and the relative response factors were expressed as the relative abundance across all analyzed samples. Reported monoacylglycerols (MAGs) are the sum of 1- and 2-acyl isomers, due to isomerization during sample processing.

\section{Fasting state assessment and sample selection}

Many of the CSF and plasma samples from AD patients were collected following additional research consent in the course of patients' clinical evaluations. Lumbar puncture procedures were nearly all scheduled in the morning, but fasting was not mandated in these individuals. Therefore, the fasting state of the samples was estimated using a previously published predictive equation [21]. A high probability of the fasted state was described by low levels of the LA-derived CYP metabolite [12(13)-
EpOME], low levels of the primary conjugated bile acid glycochenodeoxycholic acid (GCDCA), and elevated levels of the glycine-conjugated oleic acid (NO-Gly). Fasting probability was calculated using Eqs. 1 and 2.

$$
\text { Probability for fasted }=\frac{1}{(1+\operatorname{Exp}(- \text { Lin.prob.fasted }))}
$$

Equation 1. Probability of the fasted state. Where "Lin.prob.fasted" is defined by Eq. 2:

$$
\begin{aligned}
\text { Lin.prob.fasted }= & 10.01-(2.82 \times a) \\
& +(1.94 \times b)-(1.35 \times c)
\end{aligned}
$$

Equation 2. Lin. prob. fasted: $a=\log [12(13)-E p O M E] ;$ $b=\log (\mathrm{NO}-\mathrm{Gly}) ; c=\log (\mathrm{GCDCA})$. Concentrations expressed in nanomolar.

Only subjects with the probability of the fasting state > $60 \%$ were used for the plasma analysis. All subjects were used to compare lipid mediator levels in CSF. CSF was reported not to manifest postprandial lipid fluctuations [27]; additionally, comparing predicted fasted to predicted non-fasted AD subjects reveals minimal differences in only 2 metabolites (Table S2).

\section{Statistical analysis}

All statistical tests were performed using JMP Pro 14 (JMP, SAS Institute, Carry, NC). Prior to analysis, data were tested for outliers using the robust Huber $M$ test and missing data were imputed using multivariate normal imputation for variables which were at least $75 \%$ complete. The imputed numbers constitute less than 3\% of the data for both plasma and CSF. Imputation facilitated multivariate data analysis and non-imputed data were used for univariate approaches. Additionally, variables were normalized, centered, and scaled using Johnson's transformation, with normality verification using the Shapiro-Wilk test. The difference between the control and the AD group was assessed using a $t$-test with gender, age, and race as covariates. Additionally, twoway ANOVA was used to test for the gender $\times$ group and race $\times$ group interactions. In case of significant interaction, the group effect was tested separately for the interacting factor. Correlations between MoCA score and lipid mediators were assessed using Spearman's rank order correlation, to account for non-linear associations. This analysis was performed using only AD subjects, stratified by the assessed fasting state for plasma. CSF samples were analyzed without fasting state stratification. Multiple comparison control was accomplished with the false discovery rate (FDR) correction method of Benjamini and Hochberg with a $q=0.2$ [28]. 
Predictive models for AD were prepared using a combination of bootstrap tree and stepwise logistic regression modeling. Prior to analysis, subjects were randomly split into training (70\%) and validation (30\%) cohorts. Variables most frequently appearing in the models were identified by bootstrap tree: number of layers $=50$, split per tree $=3$, and bootstrap sampling rate for variables and subjects $=1$. A variable contribution scree plot was generated using variable rank and the likelihood ratio of chi-square. The scree plot was used to determine a likelihood ratio of chi-square cutoff value for variables contributing to the model. Selected variables were then subjected to stepwise logistic regressions. A stepwise analysis was performed with the maximal validation $r^{2}$ as the model stopping criteria, or if an additional step increased the Bayesian information criteria (BIC). Variables selected by the stepwise approach were then used to build the model using logistic regression. Metabolites that the model contribution $p$-value $<0.05$ were excluded, to ensure the strongest model with the minimal number of predictors.

Partial least square discriminant analysis (PLA-DA) was used to integrate AD-related differences in metabolite levels between plasma and CSF. The PLS-DA model was built using the non-linear iterative partial least squares algorithm with $\mathrm{K}$-fold variation method $(k=7)$ and included 235 variables from plasma and CSF, including metabolite levels and informative metabolite ratios. For the clarity purpose, only variables with a variable importance in projection (VIP) score $>1.4$ were displayed on the loading plot.

Correlation between CSF and plasma metabolites was assessed using Spearman's rank order correlation.

\section{Results}

\section{Fasting state assessment}

Epoxy linoleate, glycine-conjugated oleic acid, and bile acid Analysis of opportunistically collected samples brings a challenge of the unknown fasting state. The control cohort contained samples collected in the fasted state per ADRC and EHBS protocols, but the AD cohort included many who had no collected fasting state information and consist of samples collected in both fasted and non-fasted states. Therefore, to allow a direct comparison of the control and AD groups, we assessed the estimated subject fasting state using our previously published predictive model [21]. It should be noted that due to natural variation in postprandial metabolism, the fasting state predictions used here should not be considered as a binary classification (i.e., either fasted or non-fasted), but rather as a 3 group set consisting of a classical fasted profile, a classical non-fasted profile, and a mixed group of "low"-fasted profiles and "high"-non-fasted profiles that cannot be distinguished. As expected, the control group was predicted to contain mostly fasted subjects (Fig. 1). Out of 133 control subjects, 105 (i.e., 79\%) were predicted as fasted, while 17 (i.e., 12\%) as nonfasted, with a probability of $>60 \%$, and 11 (i.e., 8\%) had a fasting state probability of $<60 \%$. Out of 148 AD subjects, 60 (i.e., 40\%) were predicted as fasted and 81 (i.e., 55\%) as non-fasted, with a probability of $>60 \%$, and $7(5 \%)$ had a fasting state probability of < $60 \%$. Fifty percent of detected metabolites manifested differences between predicted fasted and non-fasted $\mathrm{AD}$ subjects in plasma and only minimal differences were observed in CSF (Table S2). These differences in plasma metabolites were in agreement with the published consensus regarding postprandial fluctuation in key metabolites, including fatty acids [29] and bile acids [30].

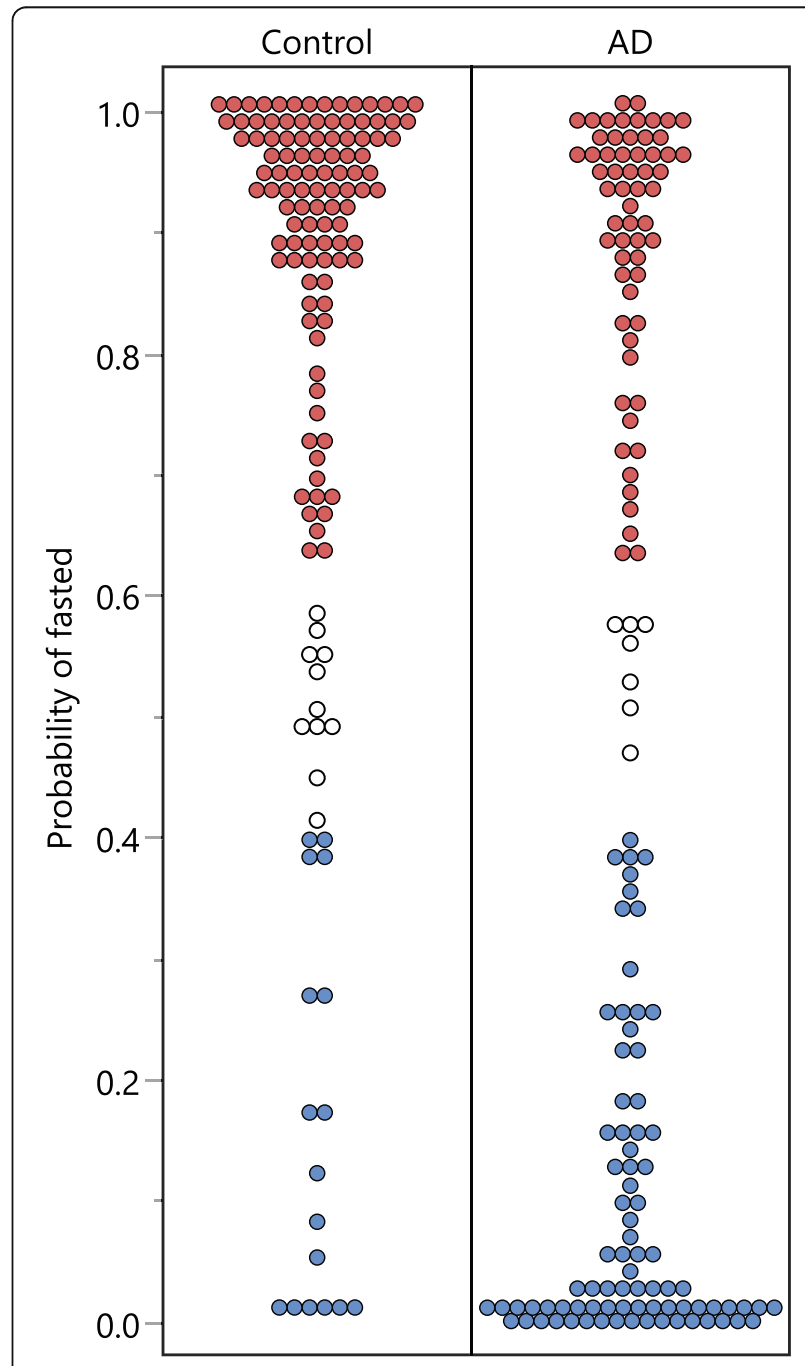

Fig. 1 Distribution of subject's probability of being in a fasted state in the control and AD groups. Colors show $60 \%$ probability cutoffs for the following: red_fasted; blue-non-fasted 
Cytochrome P450/soluble epoxide hydrolase metabolism is elevated in AD subjects

\section{Hydroxy and dihydroxy fatty acids, prostaglandins, and} fatty acid ethanolamides

We compared plasma and CSF lipid mediator concentrations between the control and the AD groups, using only estimated fasted subjects with probability $>60 \%$ for plasma. In plasma, we detected 42 oxylipins (85 measured), 5 PUFAs (5 measured), 17 endocannabinoids (22 measured), 3 NSAIDs (4 measured), 19 bile acids (23 measured), and 8 steroid hormones ( 8 measured). The mean values and $p$-values for $t$-tests and two-way ANOVA interactions for all detected metabolites are provided in Table S3. Plasma group-fold differences in the oxylipin, endocannabinoids, and PUFAs, projected onto their metabolic pathway, are presented in Fig. 2. The largest differences were observed in the long-chain omega-3 PUFA metabolism. Both EPA and DHA enzyme-derived mono-alcohols (5-LOX-derived 5-HEPE and 4-HDoHE and 12-LOX-derived 12-HEPE and 14-
$\mathrm{HDoHE}$ ) were lower (1.5-fold in average) in the AD group, when compared to the control. On the other hand, the sEH EPA metabolite 17,18-DiHETE was 3-fold higher in the $\mathrm{AD}$ group. In the $\mathrm{AD}$ group, the AA pathway manifested lower levels of the COX-derived prostaglandins PGF2 $\alpha$ and PGD2 (1.6-fold average). Additionally, the AD group showed lower levels of acylethanolamides (1.5-fold in average) derived from dihomo-gamma-linolenic acid (DGLEA), AA (AEA), docosatetraenoic acid (DEA), DHA (DHEA), and oleic acid (OEA). Notable are also lower levels of autooxidation markers, particularly the EPA-derived 9-HEPE (2fold), linoleic acid (LA)-derived TriHOMEs (1.65-fold), and AA-derived isoprostanes (1.3-fold) in the AD group.

Fewer lipid mediators were detected in CSF than in plasma. Detected CSF lipid mediators included 17 oxylipins, 5 PUFAs, 3 endocannabinoids, 14 bile acids, and 6 steroids. The mean values and $p$-values for $t$-tests and two-way ANOVA interactions are provided in Table S4. CSF significant group-fold differences in the level of

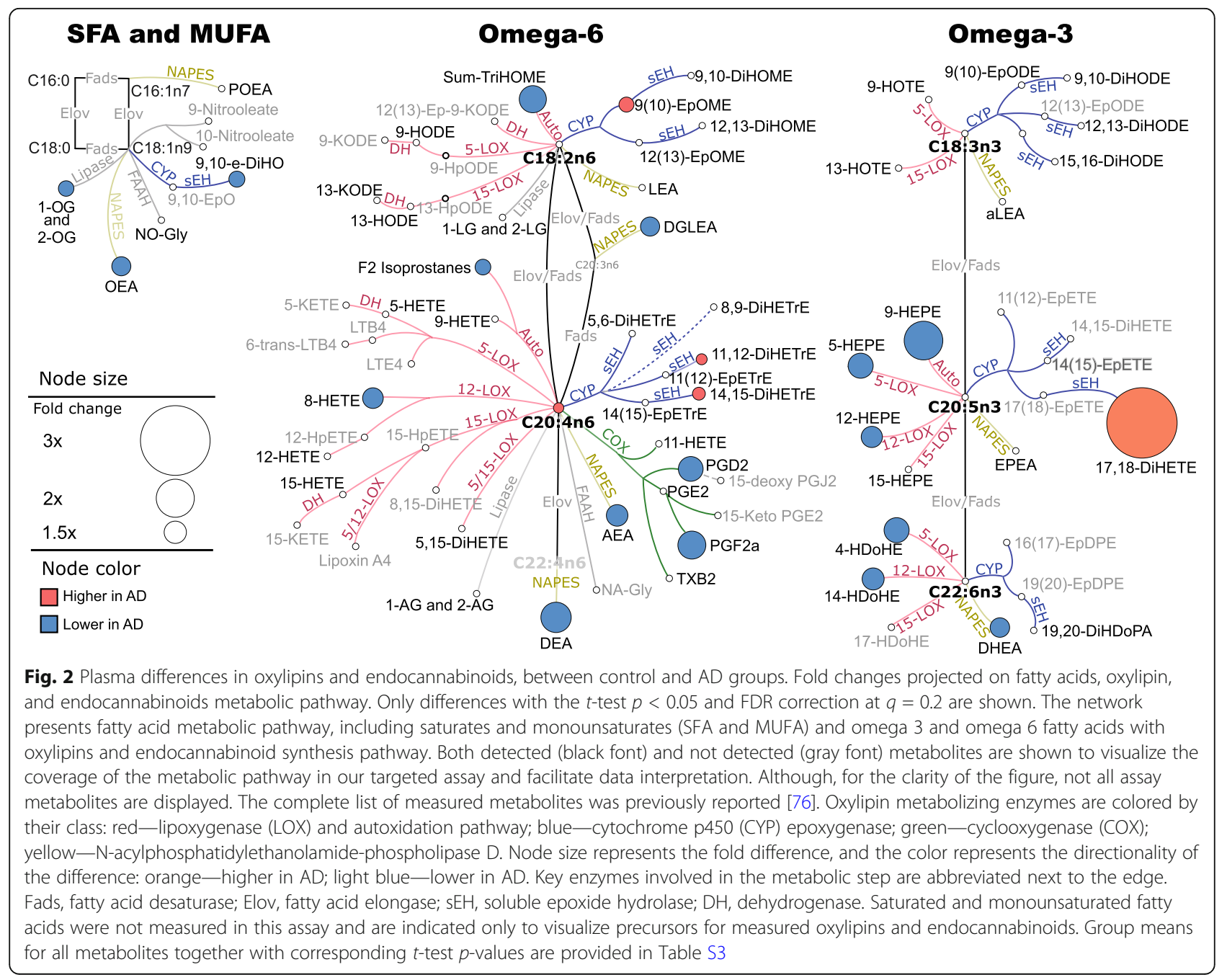


oxylipin, endocannabinoids, and PUFAs, projected onto their metabolic pathway, are presented in Fig. 3. In this matrix, the largest differences were observed in the LA CYP metabolic pathway, where both epoxy and dihydroxy FA, products of CYP and subsequent sEH metabolism, were higher in the $\mathrm{AD}$ group when compared to the control: epoxide average 1.5-fold and diol average 1.3-fold. All PUFAs from both omega-3 and omega- 6 pathways were lower in the AD group, although the difference was only 1.2-fold on average. Additionally, the AD group manifested 1.5-folds lower level of OEA and a 1.3-fold lower level of the EPA-derived 14,15-DiHETE.

\section{Bile acids}

While few differences were observed in plasma and CSF bile acid levels between control and AD subjects, numerous differences were present in the specific bile acid ratios (Table 1). Figure S1 shows the bile acid metabolic pathway together with their median plasma levels to help understand the biological aspects of specific bile acid ratios. In plasma, the $\mathrm{AD}$ group was characterized by lower levels of cholic acid (CA), a product of the neutral bile acid synthesis pathway, while chenodeoxycholic acid (CDCA), a product of the acidic pathway, was unchanged. This difference becomes even more pronounced when looking at the
CA/CDCA ratio. On the other hand, the difference between neutral and acidic pathways was not present in downstream metabolites, when comparing the secondary unconjugated bile acid ratio, like deoxycholic acid/(lithocholic acid + ursodeoxycholic acid) (DCA/ $(\mathrm{LCA}+\mathrm{UDCA}))$ or the most abundant primary conjugated derivatives glycocholic acid/glycochenodeoxycholic acid (GCA/GCDCA) (Table S3). Of note, small differences between the neutral and acidic pathways were observed in the low abundance taurine conjugates of the secondary bile acids taurodeoxycholic/ taurolithocholic acid (TDCA/TLCA). A difference in conjugation ratio (more conjugates than the substrate) was observed in the neutral synthesis pathway (GDCA/DCA and GCA/CA) but not in the acidic synthesis pathway. Differences between the neutral and acidic synthesis pathways were also observed in the conversion of the primary to secondary bile acids. The ratio of downstream products to their precursor in the neutral pathway was higher in the $\mathrm{AD}$ group in the case of DCA/CA, TDCA/CA, and GDCA/CA, but not in parallel acidic pathway metabolites (i.e., LCA/CDCA, UDCA/CDCA).

CSF manifested few differences in bile acids and their ratios. The AD group had 1.3-fold higher levels of GLCA and 1.4-fold higher level of T-a-MCA. Additionally, the

\section{SFA and MUFA Omega-6}
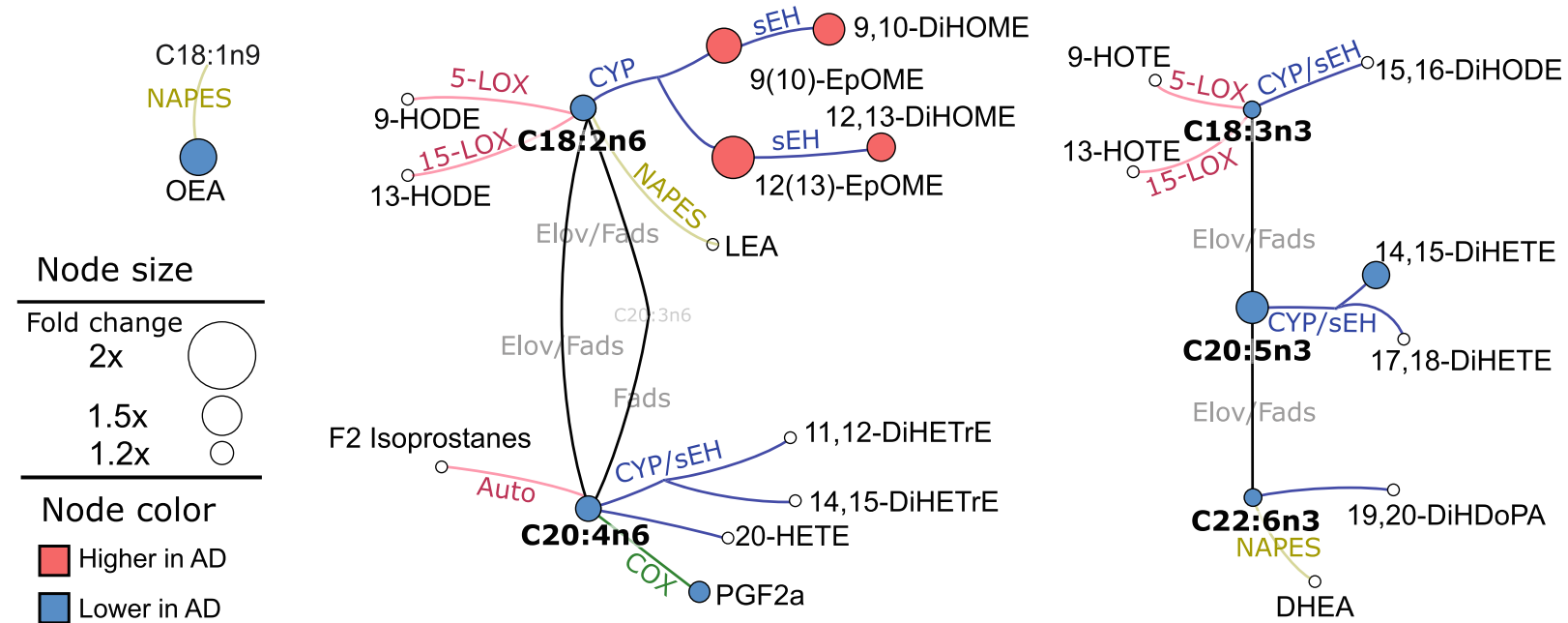

Fig. 3 CSF differences in oxylipins and endocannabinoids, between control and AD groups. Fold changes projected on fatty acids, oxylipin, and endocannabinoid metabolic pathway. Only differences with the $t$-test $p<0.05$ and FDR correction at $q=0.2$ are shown. The network presents fatty acid metabolic pathway, including saturates and monounsaturates (SFA and MUFA) and omega 3 and omega 6 fatty acids with oxylipins and endocannabinoid synthesis pathway. For clarity purposes, only metabolites detected in CSF are shown. Oxylipin metabolizing enzymes are colored by their class: red—lipoxygenase (LOX) and autoxidation pathway; blue—cytochrome p450 (CYP) epoxygenase; green—cyclooxygenase (COX); yellow- $\mathrm{N}$-acylphosphatidylethanolamide-phospholipase D. Node size represents the fold difference, and the color represents the directionality of the difference: orange-higher in AD; light blue-lower in AD. Key enzymes involved in the metabolic step are abbreviated next to the edge. Fads, fatty acid desaturase; Elov, fatty acid elongase; sEH, soluble epoxide hydrolase; DH, dehydrogenase. Saturated and monounsaturated fatty acids were not measured in this assay and are indicated only to visualize precursors for measured oxylipins and endocannabinoids. Group means for all metabolites together with corresponding $t$-test $p$-values are provided in Table S4 
Table 1 Differences in bile acid metabolites and their specific ratios between control and AD groups. Means are expressed in nanomolar or as a ratio of the concentrations. Metabolites and their ratio are stratified by the metabolic affiliations. All tested bile acids and their ratios are presented in Table S3

\begin{tabular}{|c|c|c|c|}
\hline \multirow[t]{2}{*}{ Metabolite } & \multirow{2}{*}{$\begin{array}{l}p \\
\text { value }\end{array}$} & \multicolumn{2}{|l|}{ Mean $[95 \% \mathrm{Cl}]$} \\
\hline & & Control & $A D$ \\
\hline \multicolumn{4}{|c|}{ Neutral vs acidic synthesis pathway } \\
\hline CA & 0.0321 & $27.4[20.8-36.2]$ & $19.3[13.6-27.4]$ \\
\hline CDCA & 0.978 & $38.7[29.2-51.5]$ & $46.7[32.6-66.9]$ \\
\hline CA/CDCA & 0.0008 & $0.707[0.571-0.876]$ & $0.363[0.271-0.487]$ \\
\hline GCDCA/GDCA & 0.5 & $2.08[1.66-2.61]$ & $1.97[1.51-2.57]$ \\
\hline TDCA/TLCA & 0.0114 & $1.33[1.1-1.62]$ & $1.81[1.49-2.21]$ \\
\hline \multicolumn{4}{|c|}{ Conjugation, neutral synthesis pathway } \\
\hline GDCA/DCA & 0.0031 & $0.719[0.604-0.856]$ & $1.05[0.836-1.33]$ \\
\hline TDCA/DCA & 0.1 & $0.0605[0.0467-0.0783]$ & $0.0777[0.0556-0.109]$ \\
\hline GCA/CA & 0.0104 & 2.52 [1.86-3.39] & $4.13[2.84-6.01]$ \\
\hline TCA/CA & 0.076 & $0.506[0.358-0.716]$ & $0.711[0.434-1.16]$ \\
\hline \multicolumn{4}{|c|}{ Conjugation, acidic synthesis pathway } \\
\hline GUDCA/UDCA & 0.7 & $0.546[0.341-0.876]$ & $0.515[0.334-0.793]$ \\
\hline TUDCA/UDCA & 0.746 & $2.83[1.93-4.17]$ & $2.04[1.33-3.13]$ \\
\hline GCDCA/CDCA & 0.491 & $8.15[6.21-10.7]$ & $8.36[5.68-12.3]$ \\
\hline TCDCA/CDCA & 0.644 & $0.734[0.53-1.01]$ & $0.687[0.433-1.09]$ \\
\hline TLCA/LCA & 0.961 & $0.282[0.2-0.396]$ & $0.252[0.182-0.347]$ \\
\hline GLCA/LCA & 0.721 & $0.711[0.53-0.955]$ & $0.749[0.552-1.02]$ \\
\hline \multicolumn{4}{|c|}{ Conversion of primary to secondary. Gut metagenome activity } \\
\hline $\mathrm{DCA} / \mathrm{CA}$ & 0.0459 & $8.01[5.8-11.1]$ & $12[7.89-18.1]$ \\
\hline TDCA/CA & 0.0056 & $0.469[0.309-0.711]$ & $0.906[0.517-1.59]$ \\
\hline GDCA/CA & 0.0007 & $5.76[4.01-8.27]$ & $12.5[7.71-20.2]$ \\
\hline LCA/CDCA & 0.451 & $0.66[0.419-1.04]$ & $0.589[0.42-0.825]$ \\
\hline UDCA/CDCA & 0.691 & $1.39[0.815-2.36]$ & $1.94[1.23-3.07]$ \\
\hline TUDCA & 0.0275 & $1.07[0.78-1.48]$ & $1.92[1.53-2.41]$ \\
\hline
\end{tabular}

AD group had a lower ratio of GCDCA/GLCA (1.3folds, Table S4).

\section{Steroids}

Of those measured, only a few steroid hormones showed different levels between AD and the control. In plasma, dehydroepiandrosterone sulfate (DHEAS) and progesterone were lower in the $\mathrm{AD}$ group (1.9- and 1.7-folds, respectively). Additionally, testosterone and the testosterone/progesterone ratio showed significant gender $\times$ group interaction. Female AD subjects showed 1.4-fold lower testosterone, when compared to female controls, but no differences were observed in males. On the other hand, the testosterone/progesterone ratio was 2-fold higher in $\mathrm{AD}$ male subjects compared to male controls. Testosterone/progesterone ratio differences were not observed in females.
In CSF, only corticosterone showed a significant difference between $\mathrm{AD}$ and the control group; however, the magnitude of the fold difference was only $\sim 1.1$.

\section{Relation between CSF and plasma AD markers}

In the current study, matched plasma and CSF samples were collected, allowing an assessment of the relationships between metabolites in these pools. Spearman's $\rho$ rank order correlation between plasma and CSF lipid mediator levels is shown in Table 2. The associations were distinct by metabolite classes, with oxylipins showing only 2 of 15 significant correlations, while bile acids and steroids showing 14 of 18 significant correlations. Correlations within PUFA and PUFA ethanolamide were also apparent for the long-chain omega 3 species (DHA EPA and DHA ethanolamide) but not others.

Next, we used partial least square discriminant analysis (PLS-DA) to illustrate the relationship between plasma 
Table 2 Spearman's rank order correlation between plasma and CSF metabolites. Significant $p$ values are bolded

\begin{tabular}{|c|c|c|c|}
\hline Metabolite class & Metabolite & Spearman's $\rho$ & $p$ value \\
\hline \multirow[t]{15}{*}{ Oxylipins } & PGF2a & -0.2 & 0.0746 \\
\hline & F2-IsoP & 0.34 & 0.0019 \\
\hline & 12_13-DiHOME & 0.26 & 0.0223 \\
\hline & 9_10-DiHOME & 0.15 & 0.1810 \\
\hline & 15_16-DiHODE & 0.21 & 0.0625 \\
\hline & 14_15-DiHETrE & 0.062 & 0.5830 \\
\hline & 11_12-DiHETrE & 0.11 & 0.3340 \\
\hline & 17_18-DiHETE & 0.33 & 0.0027 \\
\hline & 19_20-DiHDoPE & 0.16 & 0.1450 \\
\hline & 13-HODE & 0.029 & 0.7970 \\
\hline & 9-HODE & -0.054 & 0.6380 \\
\hline & 13-HOTE & -0.014 & 0.9010 \\
\hline & 9-HOTE & -0.051 & 0.6520 \\
\hline & 12(13)-EpOME & 0.0089 & 0.9380 \\
\hline & 9(10)-EpOME & -0.028 & 0.8070 \\
\hline \multirow[t]{3}{*}{ Acyl-EA } & OEA & -0.031 & 0.7820 \\
\hline & LEA & 0.062 & 0.5860 \\
\hline & DHEA & 0.51 & 0.0001 \\
\hline \multirow[t]{5}{*}{ PUFA } & LA & 0.15 & 0.1860 \\
\hline & ALA & 0.2 & 0.0826 \\
\hline & $\mathrm{AA}$ & -0.049 & 0.6670 \\
\hline & EPA & 0.45 & 0.0001 \\
\hline & DHA & 0.42 & 0.0001 \\
\hline \multirow[t]{12}{*}{ Bile acids } & CDCA & 0.51 & 0.0001 \\
\hline & UDCA & 0.78 & 0.0001 \\
\hline & DCA & 0.71 & 0.0001 \\
\hline & TCA & 0.62 & 0.0001 \\
\hline & TCDCA & 0.43 & 0.0001 \\
\hline & TUDCA & 0.16 & 0.1570 \\
\hline & TDCA & 0.54 & 0.0001 \\
\hline & GCA & 0.36 & 0.0012 \\
\hline & GCDCA & 0.19 & 0.0925 \\
\hline & GUDCA & 0.47 & 0.0001 \\
\hline & GDCA & 0.54 & 0.0001 \\
\hline & GLCA & -0.083 & 0.4670 \\
\hline \multirow[t]{5}{*}{ Steroids } & 17OH-PROG & 0.58 & 0.0001 \\
\hline & Cortisol & 0.3 & 0.0071 \\
\hline & Cortexolone & 0.089 & 0.4340 \\
\hline & corticosterone & 0.51 & 0.0001 \\
\hline & Testosterone & 0.81 & 0.0001 \\
\hline
\end{tabular}

and CSF AD markers (Fig. 4). That discrimination between control and $\mathrm{AD}$ was dominated by the plasma metabolites. Fifteen plasma metabolites (and their ratios) manifested variable importance in projection (VIP) score $>1.4$ compared to only 4 CSF metabolites. The discrimination between $\mathrm{AD}$ and the control group was characterized by higher plasma 17,18-DiHETE (VIP $=2.16$ ) and CSF EpOMEs (VIP = 1.95 and 1.58 for the 12(13) and 9(10) isoforms, respectively) and lower levels of the acylethanolamide ratios including both DHEA/LEA and DEA/LEA in plasma and both plasma and CSF OEA/ LEA. Plasma and CSF OEA/LEA manifest similar discriminatory power based on their proximity on the loading plot. On the other hand, plasma 17,18-DiHETE and CSF EpOMEs occupied distinct parts of the loading plot, suggesting distinct discriminatory properties. The VIPs for each metabolite are provided in Table S5.

\section{Fatty acid ethanolamides and CYP/sEH metabolites are} strong AD predictors in both plasma and CSF

We used predictive modeling to investigate how well plasma and CSF metabolites can report AD status. Plasma lipid mediators generated stronger models than those in CSF with area under the receiver operator characteristic curves (ROC AUC) of 0.924 vs. 0.824 , with the two models consisting of distinct metabolites (Fig. 5). However, in both matrices, the strongest predictors belonged to the same two metabolic pathways, the acyl ethanolamides and CYP/sEH pathway. Plasma predictors included ethanolamides (OEA and DEA normalized to the LEA level), the 12,13-DiHOME/EpOME an indicator of sEH activity [31], and sEH metabolite of AA (14,15DiHETrE). In CSF, the strongest predictors included OEA/LEA and the linoleate-derived epoxides 12(13)EpOME and 9(10)-EpOME. When plasma and CSF markers were combined in predictive model efforts, the resulting model consisted uniquely of ethanolamides, including plasma long-chain PUFA ethanolamides (DEA/ LEA and DHEA/LEA) and CSF OEA/LEA. This model resulted in the ROC AUC of 0.889 .

For all 3 models, ethanolamides OEA, DEA, and DHEA were stronger predictors when used as a ratio to LA-derived ethanolamide-LEA. LEA itself was not different between $A D$ and the control group in either plasma or CSF (Figs. 2 and 3) unlike OEA, DEA, and DHEA. Therefore, LEA likely serves as a surrogate for the general acyl ethanolamide level and adjustment of other ethanolamides by LEA lowers intra-individual variability.

To investigate the relevance of identified metabolites to the progression of $\mathrm{AD}$ pathology, components of the predictive models were applied to a linear model for $\log (\mathrm{t}-\mathrm{Tau} / \mathrm{AB} 42)$, as a marker of $\mathrm{AD}$ pathology. The $\mathrm{AD}$ predictive model components produced strong linear regression models with $\log (\mathrm{t}-\mathrm{Tau} / \mathrm{AB} 42)$ in both plasma $\left(r^{2}=0.37, p\right.$ value $\left.<0.0001\right)$ and CSF $\left(r^{2}=0.22, p\right.$ value 


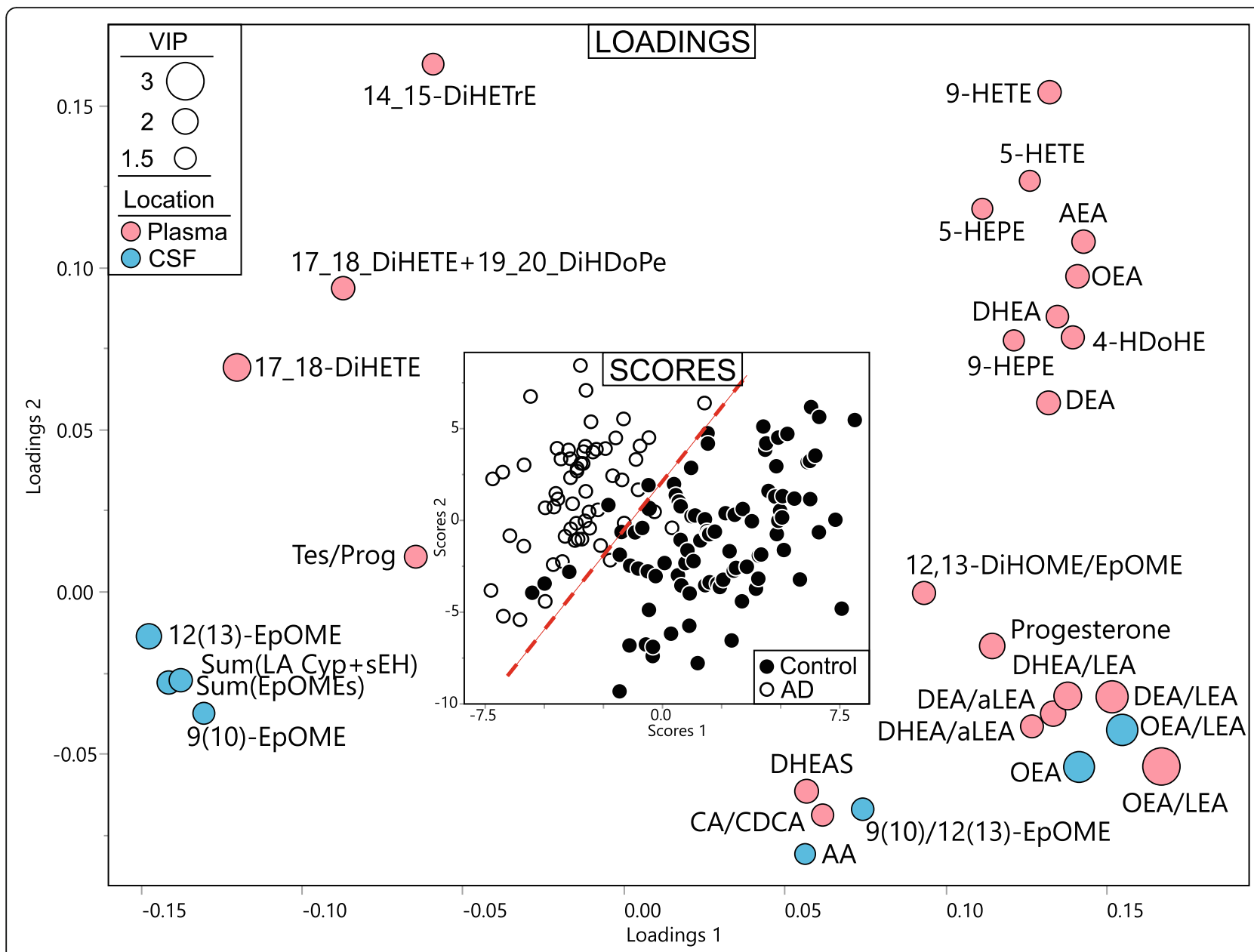

Fig. 4 Relation between plasma and CSF predictors of AD. Partial least square discriminant analysis (PLS-DA) of AD vs control, utilizing metabolites from both plasma and CSF in predicted fasted samples. Treatment group discrimination is shown by the SCORES (inset) with a plain of discrimination indicated by a dashed red line, while metabolites weighting in group discrimination are shown by the LOADINGS. Loading node color indicates metabolite origin (pink for plasma and blue for CSF). Loading node size indicates metabolite variable importance in projection (i.e., VIP). Analysis was performed with all measured metabolites, including specific ratios, but only those with VIP $\geq 1.4$ are displayed for clarity purpose

$<0.0001$ ) shown in Figure S2, further supporting our approach towards fasting state stratification.

\section{Lipid mediator-cognitive score associations in AD}

The AD cohort is characterized by a high $\log (\mathrm{t}-\mathrm{Tau} /$ A 342 ) ratio and MoCA scores ranging from normal cognitive function to severe cognitive impairment (Figure S3). Taking advantage of the broad MoCA range, we investigated lipid mediator associations with cognitive function in this group pathological levels of $t-T a u / A \beta 42$. Additionally, since the AD cohort was represented by subjects in both fasted and non-fasted states, we stratified the analysis by fasting state for plasma samples (Table 3). In the fasting state, PUFA oxidation markers, 5,15-DiHETE and 9-HETE, were negatively associated with the MoCA score (although only 5,15-DiHETE passed FDR correction). 5,15-DiHETE can have an enzymatic or autooxidative origin, whereas 9-HETE is strictly an autooxidative product. 5,15-DiIHETE correlated with 9-HETE in fasted subjects with an $R^{2}=0.415$ $(n=60 ; p<0.001)$. In non-fasted AD subjects, a strong positive association between the MoCA score and EPAderived ethanolamide (EPEA) as well as the levels of EPA and DHA were observed. Additionally, a positive correlation was detected between MoCA and the EPAderived 17,18-DiHETE, the DHA-derived 14-HDoHE, and the 18 carbon PUFAs (LA and ALA); however, these did not pass FDR correction.

In CSF, the linoleic acid-derived epoxides 12(13)- and 9(10)-EpOMEs showed weak but significant positive correlations with MoCA $(\rho>0.2, p<0.005$; Table 4). Additionally, positive associations were observed between MoCA and DHA and DHA-derived diol (19,20-DiHDoPE) and conjugated bile acids GCA (and the ratio of 


\section{Plasma}

CSF

\section{CSF and plasma}

\begin{tabular}{|c|c|c|c|c|c|c|c|c|}
\hline \multirow[b]{2}{*}{ Source } & \multirow[b]{2}{*}{ LogWorth } & \multirow[b]{2}{*}{ PValue } & \multicolumn{3}{|c|}{ Effect Summary } & \multirow[b]{2}{*}{ Source } & \multirow[b]{2}{*}{ LogWorth } & \multirow[b]{2}{*}{ PValue } \\
\hline & & & Source & LogWorth & PValue & & & \\
\hline DEA/LEA & 5.701 & 0.00000 & OEA/LEA & 5.807 & 0.00000 & Plasma DEA/LEA & 4.598 & 0.00003 \\
\hline OEA/LEA & 3.907 & 0.00011 & 12(13)-ЕрОМЕ & 4.241 & 0.00006 & CSF OEA/LEA & 3.524 & 0.00030 \\
\hline 12,13-DiHOME/ЕрОМ & IE 3.627 & 0.00024 & 9(10)-ЕрОМЕ & 2.290 & 0.00512 & Plasma DHEA/LEA & A 2.356 & 0.00441 \\
\hline 14,15-DiHETrE & 3.160 & 0.00069 & & & & & & \\
\hline
\end{tabular}

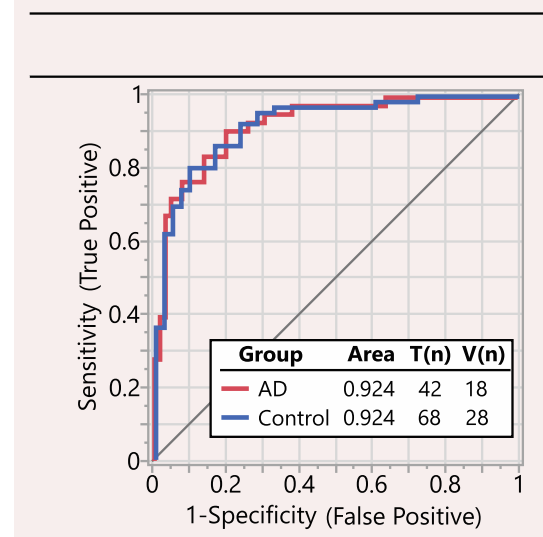

\section{Receiver Operating Characteristic}
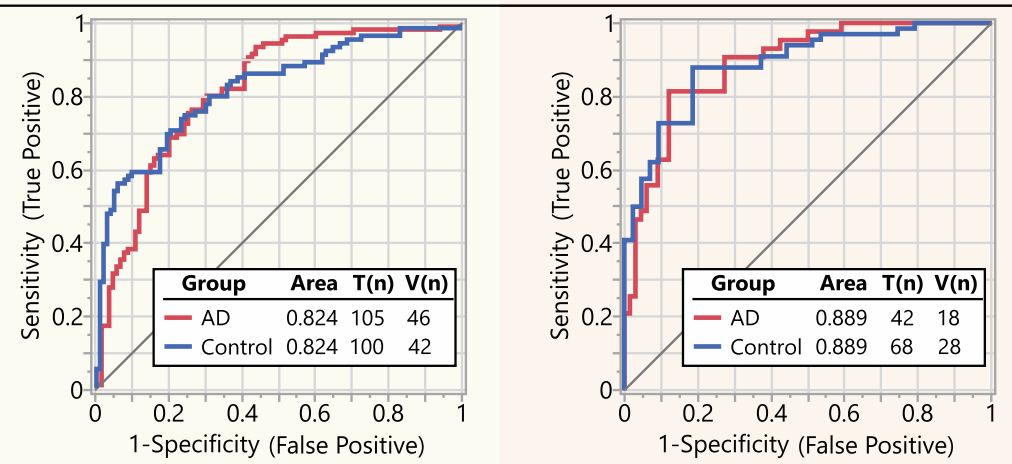

Fig. 5 Predictive model for AD with plasma and CSF metabolites. Predictive model built independently for plasma (left) and CSF (middle) and plasma + CSF (right). Effect summary shows metabolite model components, sorted by their contribution to the model, with key pathways colored in yellow (fatty acid ethanolamides) and blue (cytochrome p450/soluble epoxide hydrolase pathway). The receiver operating characteristic (ROC) curve for the training set, together with the area under the curve (AUC) and the $n$ for the training (T) and the validation $(V)$ cohorts, are showed in the bottom panel. Metabolite selection through the stepwise logistic regression model together with validation cohort parameters is provided in Table S7

GCA to GDCA and GCDCA), TCDCA, and the conjugated to unconjugated ratio for DCA and CDCA (GCA/ GCDCA, GCDCA/CDCA, and TCDCA/CDCA). However, only linoleic acid epoxides passed the FDR correction.

Additionally, utilizing subjects from both AD patients and healthy controls, we investigated associations of other components of $\mathrm{AD}$ pathology, including $\log (\mathrm{t}-$
Tau/AB42), t-Tau, AB42, p-Tau, p-Tau/t-Tau, and MoCA with plasma (Table S6) and CSF (Table S7) metabolites.

\section{Discussion}

Metabolic disruptions influencing vascular physiology, inflammation, and energy metabolism have been reported to increase the risk of Alzheimer's disease;

Table 3 Spearman's $\rho$ rank order correlation between MoCA score and plasma lipid mediators in AD patients. Analysis stratified by predicted fasted state. Only correlations with the $p<0.05$ are shown. $p$-values that passed FDR correction at $q=0.2$ are bolded

\begin{tabular}{|c|c|c|c|c|c|}
\hline \multicolumn{3}{|l|}{ Fasted } & \multicolumn{3}{|l|}{ Non-fasted } \\
\hline Metabolite & Spearman $\rho$ & $p$ value & Metabolite & Spearman $\rho$ & $p$ value \\
\hline 5,15-DiHETE & -0.448 & 0.0005 & EPEA & 0.424 & 0.0003 \\
\hline 9-HETE & -0.338 & 0.0102 & EPA & 0.386 & 0.001 \\
\hline 13-KODE & -0.299 & 0.0238 & DHA & 0.338 & 0.0043 \\
\hline \multirow[t]{6}{*}{ DCA } & -0.273 & 0.0398 & 17,18-DiHETE & 0.3 & 0.0117 \\
\hline & & & 4-HDoHE & 0.269 & 0.0246 \\
\hline & & & LA & 0.267 & 0.0254 \\
\hline & & & ALA & 0.249 & 0.0373 \\
\hline & & & 9-HETE & -0.246 & 0.0405 \\
\hline & & & 8-HETE & -0.24 & 0.0459 \\
\hline
\end{tabular}


Table 4 Spearman's $\rho$ rank order correlation between MoCA score and CSF lipid mediators in AD patients. Only correlations with the $p<0.05$ are shown. $p$-values that passed FDR correction at $q=0.2$ are bolded

\begin{tabular}{lll}
\hline Metabolite & Spearman $\boldsymbol{\rho}$ & $\boldsymbol{p}$ value \\
\hline 12(13)-EpOME & 0.279 & $\mathbf{0 . 0 0 0 9}$ \\
9(10)-EpOME & 0.24 & $\mathbf{0 . 0 0 4 7}$ \\
19,20-DiHDoPE & 0.21 & 0.0138 \\
GCA & 0.207 & 0.0152 \\
DHA & 0.203 & 0.0174 \\
GCA/GDCA & 0.202 & 0.0182 \\
GCDCA/CDCA & 0.19 & 0.026 \\
TDCA/DCA & 0.19 & 0.0261 \\
GCA/GCDCA & 0.18 & 0.0352 \\
TCDCA/CDCA & 0.172 & 0.0441 \\
TCDCA & 0.17 & 0.0468 \\
\hline
\end{tabular}

however, whether these changes are independent risk factors or how they may interact has not been well established. If novel biomarkers of $\mathrm{AD}$ can be identified within these domains, they could not only provide useful screening and risk assessment tools but may also provide insight into connections between metabolism and neurodegenerative diseases. To this end, we performed a comprehensive analysis of plasma and CSF lipid mediators and endogenous regulators of multiple processes including inflammation and energy metabolism and described their associations with $\mathrm{AD}$ and cognitive function. In the process, we identify clear differences between $\mathrm{AD}$ and healthy controls in two metabolic pathways, $\mathrm{CYP} / \mathrm{sEH}$ and fatty acid-derived ethanolamides, and subtle differences in bile acids and steroids. The potency of identified markers to predict $\mathrm{AD}$ is comparable with other plasma [32] and CSF [32] proteomic biomarkers.

$\mathrm{AD}$-associated differences in plasma bile acid were in agreement with previously reported analyses of The Religious Orders Study and the Rush Memory and Aging Project (ROS/MAP) cohort [18]. These included lower levels of $\mathrm{CA}$ and the $\mathrm{CA}: \mathrm{CDCA}$ ratio in $\mathrm{AD}$, suggesting that the neutral bile acid synthesis pathway could be affected in AD. There are few studies regarding the shift between neutral and acidic BA synthesis, one of them reporting an increase in neutral/acidic pathway product ratio in nonalcoholic steatohepatitis [33]. However, the biological relevance of this difference in terms of $\mathrm{AD}$ is yet to be determined. Interestingly, we previously reported associations between postprandial bile acids and cognition, with few associations in the fasting state [21]. Considering that the current manuscript focuses on ADrelated differences in the fasting state, further studies probing postprandial bile acid metabolism and AD are suggested. Interestingly, several bile acid and steroid differences in AD were gender specific. Few studies report gender-specific action of TUDCA and UDCA on ER stress markers in a rodent model for the prion disease [34]. These findings further support the importance of gender-focused approaches when investigating cholesterol-derived metabolism in the context of $\mathrm{AD}$, as established with regard to the links between ApoE4 and $\mathrm{AD}$ risk in post-menopausal women [35].

With respect to fatty acid metabolism, our study identified substantial $\mathrm{AD}$-associated elevations in $\mathrm{CYP} / \mathrm{sEH}$ pathway products and lower levels of acylethanolamides in both plasma and CSF, although different elements of these pathways were affected in plasma and CSF. Oxylipin and endocannabinoid levels show no association between plasma and CSF, suggesting independent regulation of these pools. Likewise, $\mathrm{CYP} / \mathrm{sEH}$ metabolites of plasma and CSF manifest distinct discriminatory power in PLS-DA models of AD. On the other hand, CSF and plasma acylethanolamides seem to manifest similar AD discriminatory power and can be substituted in the AD predictive model. These findings are consistent with previous reports implicating both $\mathrm{CYP} / \mathrm{sEH}$ metabolites and acylethanolamides as important regulators of inflammation in neurodegenerative disorders [12, 36, 37]. As some ethanolamides, like PEA, were reported to be associated with cognition in AD patients in an analysis of a small $(n=40)$ cohort [38] and sEH reports are based mainly on animal models and brain sEH gene expression [39], our study provides the first comprehensive analysis of $\mathrm{AD}$-associated alteration in the levels of the array of these lipid mediators in plasma and CSF.

In the CYP/sEH pathway, polyunsaturated fatty acids are converted to anti-inflammatory and vasodilating epoxy fatty acids by CYPs, which are further metabolized to pro-inflammatory and vasoconstricting diols by $\mathrm{sEH}$, a process primarily recognized in cardiovascular disease [40]. In the current study, we found a higher plasma level of the EPA sEH metabolite 17,18-DiHETE in AD patients, but only a slight difference in the parallel AA metabolites was observed. Notably, EPA metabolites derived from LOX pathways were lower in $\mathrm{AD}$ patients, suggesting that the observed differences are not a result of differential omega-3 fatty acid intake, but rather specific enhancement in sEH-dependent EPA metabolism. Omega-3 sEH metabolites are particularly potent regulators of the cardiovascular system, especially blood vessel tone and vascular inflammation [41] and sEH inhibitors have been suggested to improve outcomes for both cardiovascular [42] and neurodegenerative diseases [43]. Clinical associations between cardiovascular disease and $\mathrm{AD}$ have been reported, where the regulation of a vascular tone and blood flow play a role in both pathologies [44]. Additionally, we previously reported plasma EPA sEH metabolites to be negatively associated with 
perceptual speed in cognitively normal subjects [21]. Therefore, the current findings further support the involvement of vascular dysfunction in $\mathrm{AD}$, perhaps through alterations to the blood-brain barrier and vascular-related inflammatory signaling, with overlapping molecular mechanisms leading to cardiovascular and neurodegenerative pathologies. When considering these shifts in oxylipin profiles, it is important to remember that an epoxide reservoir is generated by esterification into phospholipid membranes [45], whereas diols are not readily reincorporated into the membranes and rapidly appear in the free pool and are actively excreted from cells [46]. Since tissue esterified lipid mediators were not evaluated in these samples, it is difficult to know whether the observed difference in EPA diol was due to an increased production of $\mathrm{CYP} / \mathrm{sEH}$ metabolites or increased clearance of membrane-bound EPA epoxides, and future studies are needed to resolve this issue.

In contrast to plasma, CSF showed higher levels of LA-derived epoxides, along with a moderate increase in LA diols, but not CYP/EH metabolites of longer chain PUFAs. The source of the CSF metabolites is likely tied to the central nervous system, and linoleate-derived oxylipins have been identified as the dominant form in the developing rat brain [47]. Like long-chain PUFAs, LA-derived epoxides and diols can also modulate vascular tone [48] and multiple studies point towards their cytotoxic and proinflammation nature. However, most of these studies used concentrations greatly exceeding physiological levels [49] and cytotoxic effects were sEH-dependent, pointing towards LA diols as cytotoxic agents [50]. Interestingly, LA $\mathrm{CYP} / \mathrm{sEH}$ metabolites elevated in the spinal tissue of burn victims were shown to activate the transient vanilloid receptor type 1 (TRPV1) [51]. Activation of the TRPV1 can rescue neuronal function from $A \beta$-induce impairment [52] and can alleviate cognitive and synaptic plasticity impairments in the APP23/PS45 mouse model of AD [53]. Considering that acylethanolamides are also potent activators of the TRPV1 [54], increased LA CYP metabolites may compensate for the AD-related decrease in these ethanolamides. This hypothesis is supported by positive correlation of CSF LAepoxides with the MoCA score in AD patients, suggesting elevation of epoxy fatty acids in the central nervous system being potentially beneficial in AD. It is important to mention that the transportation of oxylipins in CSF is poorly understood. In plasma, the majority of oxylipins are transported as complex lipid esters in lipoproteins, with different lipoproteins manifesting distinct oxylipin compositions [55]. Therefore, the potential for lipoprotein-dependent oxylipin transport within CSF-specific HDL particles
[56] is particularly intriguing and warrants further investigation.

Together, the CSF and plasma results implicate changes in both peripheral and central CYP/sEH metabolism in association with $\mathrm{AD}$ and cognitive impairment. These conclusions are consistent with previous reports of single nucleotide polymorphisms (SNPs) in the CYP2J2 promoter region that reduces gene expression by $\sim 50 \%$ that appears to increase the ApoE4-independent AD risk [57]. Several functional SNPs are also known to influence sEH activity and/or expression and influence disease risk [58]. Relevant to AD-associated pathologies, loss of function sEH mutations protect neurons from ischemia-induced death [59] and may alter the risk of vascular cognitive impairment [60]. Additionally, postmortem brains from human subjects with AD show higher sEH levels, when compared to the healthy controls, and sEH inhibitors can reverse microglia and astrocyte reactivity and immune pathway dysregulation in mouse $\mathrm{AD}$ models [36]. Additionally, brain sEH was positively associated with $\mathrm{AD}$ in a replicated protein-wide association study of AD [61]. Therefore, reducing sEH function appears to be protective and supports $\mathrm{sEH}$ as a valuable therapeutic target for the treatment and investigation of neuroinflammatory pathologies including AD.

Both plasma and CSF acylethanolamides were lower in $\mathrm{AD}$, with both PLS-DA and predictive model identifying OEA as the strongest predictor of $\mathrm{AD}$ in the current cohort. Acylethanolamides are generally considered anti-inflammatory [62] and neuroprotective [63] and were previously implicated in neuroinflammatory processes $[64,65]$. Their neuroprotective action is mediated by activation of the CB1 and CB2 receptors [66] and TRPV1, involved in the acute and inflammatory pain signals in the periphery [67]. Some acylethanolamides, like OEA, are also peroxisome proliferator-activated receptor (PPAR) $\alpha$ agonist [66] and regulate satiety and sleep with both central and peripheral anorexigenic effects. Notably, sleep disturbances themselves have been reported to be a risk factor for $\mathrm{AD}$ [68], and the identified reductions in CSF OEA would be consistent with such a physiological manifestation [69]. A recent study also suggested that the EPA-derived ethanolamide (EPEA) is a potential PPAR $\gamma$ agonist [70], a transcription factor known for its neuroprotective and anti-inflammatory action [71]. Interestingly, non-fasting levels of EPEA showed a positive association with MoCA in AD patients. This is in agreement with our previous findings of acylethanolamides in non-fasted individuals, including EPEA, being positively associated with perceptual speed in cognitively normal elderly individuals [21]. 
Literature provides conflicting results regarding both the levels of acylethanolamides in biological fluids, as well as the expression of CB1 and CB2 receptors in the context of $\mathrm{AD}$ [72]. Nevertheless, the body of literature suggests that exogenous cannabinoids are potent activators of the $\mathrm{CB} 1$ and $\mathrm{CB} 2$ receptors with potential therapeutic benefit for $\mathrm{AD}$ treatment, due to their neuroprotective and anti-inflammatory activity [73]. Our data suggest acylethanolamide biology is altered in relation to both $\mathrm{AD}$ pathology as well as cognition. However, future studies are needed to fully elaborate the role of these endocannabinoids in $\mathrm{AD}$ pathology.

\section{Limitations}

This study was conducted using opportunistically collected samples with the fasting state estimated using a previously developed predictive model [21] with an $\sim 17 \%$ inherent misclassification rate. Additionally, considering the small size of the AD group $(n=60)$, the finding of this study should be considered as preliminary and validated using a bigger cohort. Additionally, a small cohort prevented us from exploring the influence of factors like sex, ethnicity, and the ApoE genotype, which should be evaluated using a bigger cohort. Moreover, the results of the prediction models should be treated with caution as a small number of subjects can potentially cause overfitting.

\section{Conclusions}

In conclusion, the current study shows AD-related differences in $\mathrm{CYP} / \mathrm{sEH}$ and acylethanolamide metabolism observed in both plasma and CSF. Strong predictive and discriminant models suggest their potential as biomarkers of $\mathrm{AD}$-associated metabolic disruptions. This further supports the contention that a combination therapy reducing sEH activity with $\mathrm{sEH}$ inhibitors which have recently passed phase $1 \mathrm{a}$ trials [74], while increasing acylethanolamide tone by either exogenous supplementation or inhibiting their degradation with fatty acid amide hydrolase inhibitors [75], could be a more effective strategy than targeting either pathway independently in treating multifactorial inflammatory diseases like AD [36]. Important questions remain regarding the metabolic changes in the lipid mediators preceding pathological changes in tau and cognitive decline. We have previously reported that plasma $\mathrm{SEH}$ metabolites of the long-chain omega-3 PUFA were negatively associated and PUFA ethanolamides positively associated with perceptual speed [21], mimicking the currently described ADrelated associations. While these data suggest early alterations in these important regulatory pathways, a comprehensive analysis of longitudinal metabolome changes in relation to cognition and tauopathies is warranted. Combining assessments of dietary, lifestyle, and genetic factors promoting these metabolic changes offers the opportunity for novel risk factor discovery and the development of targeted preventive measures.

\section{Supplementary Information}

The online version contains supplementary material available at https://doi. org/10.1186/s13195-021-00893-6.

\begin{abstract}
Additional file 1 : Figure S1. Bile acids biosynthesis pathway. Node colors represent primary (blue) and secondary (dark red) bile acids. Node shape represent conjugated (diamond) and unconjugated (oval) bile acids. Node size represents median concentration in the experimental cohort. Cholesterol (top of the pathway) is converted to primary bile acids along two pathways, neutral and acidic [1]. Further, primary bile acids are secreted to gut, and portion of it being modify by the gut bacteria to secondary bile acids. Primary and secondary bile acids are reabsorbed into the blood stream and reenter the liver, where they are conjugated with amino acids glycine or taurine. Conjugated bile acids are then being secreted back to the gut along with primary bile acids. Gut bacteria can cleave conjugated amino acids off bile acids [2], and freed metabolites are recirculated. Therefore, plasma levels of conjugated bile acids can me a reflection of both liver and gut bacteria activity. Refference: 1.Pandak, W.M. and G. Kakiyama, The acidic pathway of bile acid synthesis: Not just an alternative pathway(). Liver Res, 2019. 3(2): p. 88-98; 2. Ridlon, J.M., et al., Consequences of bile salt biotransformations by intestinal bacteria. Gut Microbes, 2016. 7(1): p. 22-39.
\end{abstract}

Additional file $\mathbf{2}$ : Figure S2. Multilinear regression of $\log (\mathrm{t}-\mathrm{Tau} / \mathrm{AB} 42)$ and the components of AD predictive models, presented in the Figure 4. Analysis performed separately for plasma (upper panel) and CSF (lower panel) AD predictors. Association of individual components are shown in the leverage plots, whereas effect summery contain descriptive statistics for each individual metabolite in the model.

Additional file 3 : Figure S3. Control and AD group MoCA and log(tTau/Aß42).

Additional file 4 : Table S1. Cohort Characteristics.

Additional file $\mathbf{5}$ : Table S2. T-test of predicted fasted vs predicted non-fasted in AD group for plasma and CSF metabolites.

Additional file $\mathbf{6}$ : Table $\mathbf{5 3}$. The mean values and t-test and two-way ANOVA interaction $p$-values for all detected metabolites in plasma.

Additional file $\mathbf{7}$ : Table S4. The mean values and t-test and two-way ANOVA interaction $p$-values for all detected metabolites in CSF. P-values $<0.05$ are colored red, $p$-values $>0.05<0.1$ are colored orange.

Additional file 8 : Table S5. Variable importance in projection (VIP) scores for all plasma and CSF variables used for partial least square discriminant analysis (PLS-DA).

Additional file 9 : Table S6. Spearman's rank order correlation between AD-related markers and plasma lipid mediators. Analysis performed utilizing only subjects predicted to be fasted with the probability $>60 \%$, including $60 \mathrm{AD}$ patients and 96 healthy controls. Only associations with the $p<0.05$ are displayed. Associations colored based on Spearman's $\rho$ (values shown in the table), with blue color for negative and orange for positive associations.

Additional file 10 : Table S7. Spearman's rank order correlation between AD-related markers and CSF lipid mediators. Analysis performed utilizing 151 AD patients and 142 healthy controls. Only associations with the $p>0.05$ are displayed. Associations colored based on Spearman's $\rho$ (values shown in the table), with blue color for negative and orange for positive associations.

Additional file 11 : Table S8. Stepwise logistic model predicting AD status using plasma, CSF or both plasma and CSF metabolites. Stepwise analysis was performed with the maximal validation $r^{2}$ as the model 
stopping criteria, or if an additional step increased the BIC. Model stopping point for each analysis is highlighted.

\section{Acknowledgements}

The investigators within the ADMC, not listed specifically in this publication's author's list, provided data but did not participate in the analysis or writing of this manuscript. A complete listing of ADMC investigators can be found at https://sites.duke.edu/adnimetab/team/.

\section{Authors' contributions}

$\mathrm{KB}$, JWN, and TLP contributed to metabolomic data generation and quality assessment and quality control. KB and JWN analyzed the data and wrote the manuscript. RKD, NTS, J L, AlL, and CMH designed the study and contributed to data interpretation. EBD contributed to data interpretation. NTS, JJL, AlL, GL, and CB contributed to the subject selection and collected the clinical data and the samples. RKD, NTS, JJL, and AlL revised the manuscript. All authors read and approved the final manuscript.

\section{Funding}

The Alzheimer's Disease Metabolomics Consortium (ADMC) is funded wholly or in part by the following National Institute on Aging grants and supplements, components of the Accelerating Medicines Partnership for AD (AMP-AD) and/or Molecular Mechanisms of the Vascular Etiology of AD (M2OVE-AD): NIA R01AG046171, RF1AG051550, RF1AG057452, R01AG059093, RF1AG058942, U01AG061359, U19AG063744, and FNIH: \#DAOU16AMPA awarded to Dr. Kaddurah-Daouk at Duke University in partnership with a large number of academic institutions. Additional support was provided by Emory ADRC P30 AG066511 awarded to Allan I. Levey, Emory EHBS R01 AG070937 awarded to James J. Lah, and USDA Intramural Projects 203251530-022-00D and 2032-51530-025-00D awarded to John W. Newman. The USDA is an equal opportunity employer and provider.

\section{Availability of data and materials}

Metabolomics data is provided by the Alzheimer's Disease Metabolomics Consortium (ADMC). Metabolomics data and pre-processed data are accessible through the Accelerating Medicines Partnership for AD (AMP-AD) Knowledge Portal (https://ampadportal.org). The AMP-AD Knowledge Portal is the distribution site for data, analysis results, analytical methodology, and research tools generated by the AMP-AD Target Discovery and Preclinical Validation Consortium and multiple Consortia and research programs supported by the National Institute on Aging.

\section{Declarations}

\section{Ethics approval and consent to participate}

All participants from whom plasma and CSF samples were collected provided informed consent under protocols approved by the Institutional Review Board at Emory University. Cohorts included the Emory Healthy Brain Study (IRB00080300), Cognitive Neurology Research (IRB00078273), and Memory @ Emory (IRB00079069). All protocols were reviewed and approved by the Emory University Institutional Review Board. Ethics approval for metabolomics analysis: Analysis samples shipped from Emory were approved for metabolomic profiling and data analysis by the Institutional Review Board at Duke University (Pro00079616).

\section{Consent for publication}

Not applicable.

\section{Competing interests}

The authors declare that they have no competing interests. Dr. KaddurahDaouk is an inventor on several patents in the field of metabolomics and holds equity in Metabolon Inc. which was not involved in this study.

\section{Author details}

'West Coast Metabolomics Center, Genome Center, University of California Davis, Davis, CA 95616, USA. ²Department of Food Science and Technology, University of California - Davis, Davis, CA 95616, USA. ${ }^{3}$ Department of Biochemistry, Emory University School of Medicine, Atlanta, GA 30322, USA. ${ }^{4}$ Department of Neurology, Emory University, Atlanta, GA 30329, USA. ${ }^{5}$ Duke Molecular Physiology Institute, Duke University, Durham, NC 27708, USA.
${ }^{6}$ Department of Psychiatry and Behavioral Sciences, Duke University, Durham, NC 27708, USA. 'Department of Psychiatry and Behavioral Sciences, Duke Institute for Brain Sciences and Department of Medicine, Duke University, Durham, NC 27708, USA. ${ }^{8}$ Western Human Nutrition Research Center, United States Department of Agriculture - Agriculture Research Service, Davis, CA 95616, USA. ${ }^{9}$ Department of Nutrition, University of California - Davis, Davis, CA 95616, USA.

Received: 10 March 2021 Accepted: 25 August 2021

Published online: 06 September 2021

\section{References}

1. Alkan E, Taporoski TP, Sterr A, von Schantz M, Vallada H, Krieger JE, et al. Metabolic syndrome alters relationships between cardiometabolic variables, cognition and white matter hyperintensity load. Sci Rep. 2019;9(1):4356. https://doi.org/10.1038/s41598-019-40630-6.

2. Bosia M, Buonocore M, Bechi M, Santarelli L, Spangaro M, Cocchi F, et al. Improving cognition to increase treatment efficacy in schizophrenia: effects of metabolic syndrome on cognitive remediation's outcome. Front Psychiatry. 2018;9:647. https://doi.org/10.3389/fpsyt.2018.00647.

3. Monthe-Dreze C, Rifas-Shiman SL, Gold DR, Oken E, Sen S. Maternal obesity and offspring cognition: the role of inflammation. Pediatr Res. 2019;85(6): 799-806. https://doi.org/10.1038/s41390-018-0229-z.

4. Gabbs M, Leng S, Devassy JG, Monirujiaman M, Aukema HM. Advances in our understanding of oxylipins derived from dietary PUFAs. Adv Nutr. 2015; 6(5):513-40. https://doi.org/10.3945/an.114.007732.

5. Nayeem MA. Role of oxylipins in cardiovascular diseases. Acta Pharmacol Sin. 2018;39(7):1142-54. https://doi.org/10.1038/aps.2018.24.

6. Huang CC, Chang MT, Leu HB, Yin WH, Tseng WK, Wu YW, et al. Association of arachidonic acid-derived lipid mediators with subsequent onset of acute myocardial infarction in patients with coronary artery disease. Sci Rep. 2020; 10(1):8105. https://doi.org/10.1038/s41598-020-65014-z.

7. Ma H, Patti ME. Bile acids, obesity, and the metabolic syndrome. Best Pract Res Clin Gastroenterol. 2014;28(4):573-83. https://doi.org/10.1016/j.bpg.2014 07.004.

8. Serhan CN. Pro-resolving lipid mediators are leads for resolution physiology. Nature. 2014;510(7503):92-101. https://doi.org/10.1038/nature13479.

9. Schimke I, Griesmacher A, Weigel G, Holzhutter HG, Muller MM. Effects of reactive oxygen species on eicosanoid metabolism in human endothelial cells. Prostaglandins. 1992;43(3):281-92. https://doi.org/10.1016/00906980(92)90096-C.

10. Bellocchio L, Cervino C, Pasquali R, Pagotto U. The endocannabinoid system and energy metabolism. J Neuroendocrinol. 2008;20(6):850-7. https://doi. org/10.1111/j.1365-2826.2008.01728.x.

11. Zou S, Kumar U. Cannabinoid receptors and the endocannabinoid system: signaling and function in the central nervous system.Int J Mol Sci. 2018; 19(3):833.

12. Chiurchiu V, van der Stelt M, Centonze D, Maccarrone M. The endocannabinoid system and its therapeutic exploitation in multiple sclerosis: clues for other neuroinflammatory diseases. Prog Neurobiol. 2018; 160:82-100. https://doi.org/10.1016/j.pneurobio.2017.10.007.

13. Chiang JY. Bile acid metabolism and signaling. Compr Physiol. 2013;3(3): 1191-212. https://doi.org/10.1002/cphy.c120023.

14. Guo C, Chen WD, Wang YD. TGR5, not only a metabolic regulator. Front Physiol. 2016;7:646.

15. Bazan NG, Colangelo V, Lukiw WJ. Prostaglandins and other lipid mediators in Alzheimer's disease. Prostaglandins Other Lipid Mediat. 2002;68-69:197210.

16. Kao YC, Ho PC, Tu YK, Jou IM, Tsai KJ. Lipids and Alzheimer's disease. Int J Mol Sci. 2020;21(4):1505

17. Miyazawa K, Fukunaga H, Tatewaki Y, Takano Y, Yamamoto S, Mutoh T, et al. Alzheimer's disease and specialized pro-resolving lipid mediators: do MaR1, RvD1, and NPD1 show promise for prevention and treatment? Int J Mol Sci. 2020;21(16):5783.

18. MahmoudianDehkordi S, Arnold M, Nho K, Ahmad S, Jia W, Xie G, et al. Altered bile acid profile associates with cognitive impairment in Alzheimer's disease-an emerging role for gut microbiome. Alzheimers Dement. 2019; 15(1):76-92. https://doi.org/10.1016/j.jalz.2018.07.217.

19. Baloni P, Funk CC, Yan J, Yurkovich JT, Kueider-Paisley A, Nho K, et al. Metabolic network analysis reveals altered bile acid synthesis and 
metabolism in Alzheimer's disease. Cell Rep Med. 2020;1 (8):100138. https:// doi.org/10.1016/j.xcrm.2020.100138

20. Ackerman HD, Gerhard GS. Bile acids in neurodegenerative disorders. Front Aging Neurosci. 2016;8:263.

21. Borkowski K, Taha AY, Pedersen TL, De Jager PL, Bennett DA, KaddurahDaouk R, et al. Serum metabolomic biomarkers of perceptual speed in cognitively normal and mildly impaired subjects with fasting state stratification. BioRxiv. . https://doi.org/10.1101/2020.09.03.282343.

22. Goetz ME, Hanfelt JJ, John SE, Bergquist SH, Loring DW, Quyyumi A, et al. Rationale and design of the Emory Healthy Aging and Emory Healthy Brain Studies. Neuroepidemiology. 2019;53(3-4):187-200. https://doi.org/10.1159/ 000501856.

23. Shaw LM, Vanderstichele H, Knapik-Czajka M, Clark CM, Aisen PS, Petersen RC, et al. Cerebrospinal fluid biomarker signature in Alzheimer's disease neuroimaging initiative subjects. Ann Neurol. 2009;65(4):403-13. https://doi. org/10.1002/ana.21610.

24. Hulstaert F, Blennow K, Ivanoiu A, Schoonderwaldt HC, Riemenschneider M, De Deyn PP, et al. Improved discrimination of AD patients using betaamyloid(1-42) and tau levels in CSF. Neurology. 1999;52(8):1555-62. https:// doi.org/10.1212/WNL.52.8.1555.

25. Pedersen TL, Gray IJ, Newman JW. Plasma and serum oxylipin, endocannabinoid, bile acid, steroid, fatty acid and nonsteroidal antiinflammatory drug quantification in a 96-well plate format. Anal Chim Acta. 2021;1143:189-200. https://doi.org/10.1016/j.aca.2020.11.019.

26. Agrawal K, Hassoun LA, Foolad N, Pedersen TL, Sivamani RK, Newman JW. Sweat lipid mediator profiling: a noninvasive approach for cutaneous research. J Lipid Res. 2017;58(1):188-95. https://doi.org/10.1194/jlr.M071738.

27. Saito K, Hattori K, Andou T, Satomi Y, Gotou M, Kobayashi H, et al. Characterization of postprandial effects on CSF metabolomics: a pilot study with parallel comparison to plasma.Metabolites. 2020;10(5):185.

28. Benjamini $Y$, Hochberg $Y$. Controlling the false discovery rate: a practical and powerful approach to multiple testing. J Royal Stat Soc Ser B (Methodological). 1995;57(1):289-300. https://doi.org/10.1111/j.2517-6161.1 995.tb02031.x.

29. Normand-Lauziere F, Frisch F, Labbe SM, Bherer P, Gagnon R, Cunnane SC, et al. Increased postprandial nonesterified fatty acid appearance and oxidation in type 2 diabetes is not fully established in offspring of diabetic subjects. PLoS One. 2010;5(6):e10956. https://doi.org/10.1371/journal.pone. 0010956.

30. Fiamoncini J, Yiorkas AM, Gedrich K, Rundle M, Alsters SI, Roeselers G, et al. Determinants of postprandial plasma bile acid kinetics in human volunteers. Am J Physiol Gastrointest Liver Physiol. 2017;313(4):G300-G12. https://doi. org/10.1152/ajpgi.00157.2017.

31. Lee $C R$, North KE, Bray MS, Fornage M, Seubert JM, Newman JW, et al. Genetic variation in soluble epoxide hydrolase (EPHX2) and risk of coronary heart disease: the Atherosclerosis Risk in Communities (ARIC) study. Hum Mol Genet. 2006;15(10):1640-9. https://doi.org/10.1093/hmg/ ddl085.

32. Palmqvist $\mathrm{S}$, Tideman $\mathrm{P}$, Cullen N, Zetterberg H, Blennow K, Alzheimer's Disease Neuroimaging I, et al. Prediction of future Alzheimer's disease dementia using plasma phospho-tau combined with other accessible measures. Nat Med. 2021;27:1034-42.

33. Jahn D, Geier A. Bile acids in nonalcoholic steatohepatitis: pathophysiological driving force or innocent bystanders? Hepatology. 2018; 67(2):464-6. https://doi.org/10.1002/hep.29543.

34. Grant SM, DeMorrow S. Bile acid signaling in neurodegenerative and neurological disorders. Int J Mol Sci. 2020;21(17):5982.

35. Neu SC, Pa J, Kukull W, Beekly D, Kuzma A, Gangadharan P, et al. Apolipoprotein E genotype and sex risk factors for Alzheimer disease: a meta-analysis. JAMA Neurol. 2017;74(10):1178-89. https://doi.org/10.1001/ja maneurol.2017.2188.

36. Ghosh A, Comerota ME, Wan D, Chen F, Propson NE, Hwang SH, et al. Epoxy fatty acid dysregulation and neuroinflammation in Alzheimer's disease is resolved by a soluble epoxide hydrolase inhibitor. BioRxiv. . https://doi.org/10.1101/2020.06.30.180984.

37. Kodani SD, Morisseau C. Role of epoxy-fatty acids and epoxide hydrolases in the pathology of neuro-inflammation. Biochimie. 2019;159:59-65. https:// doi.org/10.1016/j.biochi.2019.01.020

38. Altamura C, Ventriglia M, Martini MG, Montesano D, Errante $Y$, Piscitelli F, et al. Elevation of plasma 2-arachidonoylglycerol levels in Alzheimer's disease patients as a potential protective mechanism against neurodegenerative decline. J Alzheimers Dis. 2015;46(2):497-506. https://doi. org/10.3233/JAD-142349

39. Grinan-Ferre C, Codony S, Pujol E, Yang J, Leiva R, Escolano C, et al. Pharmacological inhibition of soluble epoxide hydrolase as a new therapy for Alzheimer's disease. Neurotherapeutics. 2020;17(4):1825-35. https://doi. org/10.1007/s13311-020-00854-1.

40. Deng Y, Theken KN, Lee CR. Cytochrome P450 epoxygenases, soluble epoxide hydrolase, and the regulation of cardiovascular inflammation. J Mol Cell Cardiol. 2010;48(2):331-41. https://doi.org/10.1016/j.yjmcc.2009.10.022.

41. Ulu A, Harris TR, Morisseau C, Miyabe C, Inoue H, Schuster G, et al. Antiinflammatory effects of omega-3 polyunsaturated fatty acids and soluble epoxide hydrolase inhibitors in angiotensin-II-dependent hypertension. J Cardiovasc Pharmacol. 2013;62(3):285-97. https://doi.org/10.1097/FJC.0b013 e318298e460

42. Imig JD, Hammock BD. Soluble epoxide hydrolase as a therapeutic target for cardiovascular diseases. Nat Rev Drug Discov. 2009;8(10):794-805. https://doi.org/10.1038/nrd2875.

43. Wagner KM, McReynolds CB, Schmidt WK, Hammock BD. Soluble epoxide hydrolase as a therapeutic target for pain, inflammatory and neurodegenerative diseases. Pharmacol Ther. 2017;180:62-76. https://doi. org/10.1016/j.pharmthera.2017.06.006.

44. Firoz CK, Jabir NR, Khan MS, Mahmoud M, Shakil S, Damanhouri GA, et al. An overview on the correlation of neurological disorders with cardiovascular disease. Saudi J Biol Sci. 2015;22(1):19-23. https://doi.org/10.1 016/j.sjbs.2014.09.003.

45. Klett EL, Chen S, Edin ML, Li LO, Ilkayeva O, Zeldin DC, et al. Diminished acyl-CoA synthetase isoform 4 activity in INS 832/13 cells reduces cellular epoxyeicosatrienoic acid levels and results in impaired glucose-stimulated insulin secretion. J Biol Chem. 2013;288(30):21618-29. https://doi.org/10.1 074/jbc.M113.481077.

46. Greene JF, Williamson KC, Newman JW, Morisseau C, Hammock BD. Metabolism of monoepoxides of methyl linoleate: bioactivation and detoxification. Arch Biochem Biophys. 2000;376(2):420-32. https://doi.org/1 $0.1006 / a b b i .2000 .1753$

47. Hennebelle M, Morgan RK, Sethi S, Zhang Z, Chen H, Grodzki AC, et al. Linoleic acid-derived metabolites constitute the majority of oxylipins in the rat pup brain and stimulate axonal growth in primary rat cortical neuronglia co-cultures in a sex-dependent manner. J Neurochem. 2020;152(2):195207. https://doi.org/10.1111/jnc.14818.

48. Takahashi H, Ishizaki T, Ameshima S, Kishi Y, Sasaki F, Nakai T, et al. Leukotoxin, 9,10-epoxy-12-octadecenoate, causes vasodilation in isolated pulmonary artery rings preconstricted with endothelin 1. Nihon Kyobu Shikkan Gakkai Zasshi. 1992;30(3):418-24.

49. Hildreth K, Kodani SD, Hammock BD, Zhao L. Cytochrome P450-derived linoleic acid metabolites EpOMEs and DiHOMEs: a review of recent studies. J Nutr Biochem. 2020;86:108484. https://doi.org/10.1016/j. jnutbio.2020.108484.

50. Moghaddam MF, Grant DF, Cheek JM, Greene JF, Williamson KC, Hammock BD. Bioactivation of leukotoxins to their toxic diols by epoxide hydrolase. Nat Med. 1997;3(5):562-6. https://doi.org/10.1038/nm0597-562.

51. Green D, Ruparel S, Gao X, Ruparel N, Patil M, Akopian A, et al. Central activation of TRPV1 and TRPA1 by novel endogenous agonists contributes to mechanical allodynia and thermal hyperalgesia after burn injury. Mol Pain. 2016;12:174480691666172. https://doi.org/10.1177/1744 806916661725

52. Balleza-Tapia H, Crux S, Andrade-Talavera Y, Dolz-Gaiton P, Papadia D, Chen $G$, et al. TrpV1 receptor activation rescues neuronal function and network gamma oscillations from Abeta-induced impairment in mouse hippocampus in vitro. Elife. 2018;7. https://doi.org/10.7554/eLife.37703.

53. Du Y, Fu M, Huang Z, Tian X, Li J, Pang Y, et al. TRPV1 activation alleviates cognitive and synaptic plasticity impairments through inhibiting AMPAR endocytosis in APP23/PS45 mouse model of Alzheimer's disease. Aging Cell. 2020;19(3):e13113. https://doi.org/10.1111/acel.13113.

54. Raboune S, Stuart JM, Leishman E, Takacs SM, Rhodes B, Basnet A, et al. Novel endogenous $\mathrm{N}$-acyl amides activate TRPV1-4 receptors, BV-2 microglia, and are regulated in brain in an acute model of inflammation. Front Cell Neurosci. 2014:8:195

55. Borkowski K, Yim SJ, Holt RR, Hackman RM, Keen CL, Newman JW, et al. Walnuts change lipoprotein composition suppressing TNFalpha-stimulated cytokine production by diabetic adipocyte. J Nutr Biochem. 2019;68:51-8. https://doi.org/10.1016/j.jnutbio.2019.03.004. 
56. Mahley RW. Central nervous system lipoproteins: ApoE and regulation of cholesterol metabolism. Arterioscler Thromb Vasc Biol. 2016;36(7):1305-15. https://doi.org/10.1161/ATVBAHA.116.307023.

57. Yan HC, Kong YY, He BX, Huang MK, Li J, Zheng JQ, et al. CYP2J2 rs890293 polymorphism is associated with susceptibility to Alzheimer's disease in the Chinese Han population. Neuroscience Letters. 2015;593:56-60. https://doi. org/10.1016/j.neulet.2015.03.024.

58. Srivastava PK, Sharma VK, Kalonia DS, Grant DF. Polymorphisms in human soluble epoxide hydrolase: effects on enzyme activity, enzyme stability, and quaternary structure. Arch Biochem Biophys. 2004;427(2):164-9. https://doi. org/10.1016/j.abb.2004.05.003.

59. Koerner IP, Jacks R, DeBarber AE, Koop D, Mao P, Grant DF, et al. Polymorphisms in the human soluble epoxide hydrolase gene EPHX2 linked to neuronal survival after ischemic injury. J Neurosci. 2007;27(17):4642-9. https://doi.org/10.1523/JNEUROSCI.0056-07.2007.

60. Nelson JW, Young JM, Borkar RN, Woltjer RL, Quinn JF, Silbert LC, et al. Role of soluble epoxide hydrolase in age-related vascular cognitive decline. Prostaglandins Other Lipid Mediat. 2014;113-115:30-7.

61. Wingo AP, Liu Y, Gerasimov ES, Gockley J, Logsdon BA, Duong DM, et al. Integrating human brain proteomes with genome-wide association data implicates new proteins in Alzheimer's disease pathogenesis. Nat Genet. 2021;53(2):143-6. https://doi.org/10.1038/s41 588-020-00773-z.

62. Turcotte C, Chouinard F, Lefebvre JS, Flamand N. Regulation of inflammation by cannabinoids, the endocannabinoids 2-arachidonoylglycerol and arachidonoyl-ethanolamide, and their metabolites. J Leukoc Biol. 2015;97(6):1049-70. https://doi.org/10.1189/jlb.3RU0115021R.

63. Petrosino S, Di Marzo V. The pharmacology of palmitoylethanolamide and first data on the therapeutic efficacy of some of its new formulations. Br J Pharmacol. 2017;174(11):1349-65. https://doi.org/1 $0.1111 / \mathrm{bph} .13580$

64. Saito VM, Rezende RM, Teixeira AL. Cannabinoid modulation of neuroinflammatory disorders. Curr Neuropharmacol. 2012;10(2):159-66. https://doi.org/10.2174/157015912800604515.

65. Bisogno T, Di Marzo V. The role of the endocannabinoid system in Alzheimer's disease: facts and hypotheses. Curr Pharm Des. 2008;14(23): 2299-3305. https://doi.org/10.2174/138161208785740027.

66. Bradshaw HB, Walker JM. The expanding field of cannabimimetic and related lipid mediators. Br J Pharmacol. 2005;144(4):459-65. https://doi.org/1 0.1038/sj.bjp.0706093.

67. Bradshaw HB, Raboune S, Hollis JL. Opportunistic activation of TRP receptors by endogenous lipids: exploiting lipidomics to understand TRP receptor cellular communication. Life Sci. 2013;92(8-9):404-9. https://doi.org/10.1016/ j.lfs.2012.11.008.

68. Li K, Luo X, Zeng Q, Jiaerken Y, Wang S, Xu X, et al. Interactions between sleep disturbances and Alzheimer's disease on brain function: a preliminary study combining the static and dynamic functional MRI. Sci Rep. 2019;9(1): 19064. https://doi.org/10.1038/s41598-019-55452-9.

69. Koethe D, Schreiber D, Giuffrida A, Mauss C, Faulhaber J, Heydenreich B, et al. Sleep deprivation increases oleoylethanolamide in human cerebrospinal fluid. J Neural Transm (Vienna). 2009;116(3):301-5. https://doi. org/10.1007/s00702-008-0169-6

70. Giordano C, Plastina P, Barone I, Catalano S, Bonofiglio D. n-3 polyunsaturated fatty acid amides: new avenues in the prevention and treatment of breast cancer. Int J Mol Sci. 2020;21(7):2279.

71. Villapol S. Roles of peroxisome proliferator-activated receptor gamma on brain and peripheral inflammation. Cell Mol Neurobiol. 2018;38(1):121-32. https://doi.org/10.1007/s10571-017-0554-5.

72. Berry AJ, Zubko O, Reeves SJ, Howard RJ. Endocannabinoid system alterations in Alzheimer's disease: a systematic review of human studies. Brain Res. 2020;1749:147135.

73. Aso E, Ferrer I. Cannabinoids for treatment of Alzheimer's disease: moving toward the clinic. Front Pharmacol. 2014;5:37.

74. Hammock BD, McReynolds CB, Wagner K, Buckpitt A, Cortes-Puch I, Croston $\mathrm{G}$, et al. Movement to the clinic of soluble epoxide hydrolase inhibitor EC5026 as an analgesic for neuropathic pain and for use as a nonaddictive opioid alternative. J Med Chem. 2021;64(4):1856-72. https://doi.org/10.1 021/acs.jmedchem.0c01886.

75. Booker L, Kinsey SG, Abdullah RA, Blankman JL, Long JZ, Ezzili C, et al. The fatty acid amide hydrolase (FAAH) inhibitor PF-3845 acts in the nervous system to reverse LPS-induced tactile allodynia in mice. $\mathrm{Br}$ J Pharmacol. 2012;165(8):2485-96. https://doi.org/10.1111/j.1476-5381.2011. 01445.x.

76. Pedersen TL, Newman JW. Establishing and performing targeted multiresidue analysis for lipid mediators and fatty acids in small clinical plasma samples. Methods Mol Biol. 2018;1730:175-212.

\section{Publisher's Note}

Springer Nature remains neutral with regard to jurisdictional claims in published maps and institutional affiliations.
Ready to submit your research? Choose BMC and benefit from:

- fast, convenient online submission

- thorough peer review by experienced researchers in your field

- rapid publication on acceptance

- support for research data, including large and complex data types

- gold Open Access which fosters wider collaboration and increased citations

- maximum visibility for your research: over $100 \mathrm{M}$ website views per year

At BMC, research is always in progress.

Learn more biomedcentral.com/submissions 